\title{
Introduction to telemedicine. Second edition
}

\author{
Edited by Richard Wootton, John Craig, Victor Patterson \\ London: The Royal Society of Medicine \\ Press Ltd, 2006, pp. 206, \\ ISBN 1853156779
}

This book provides an introduction to telemedicine, a phenomenon defined by the authors as "rapid access to shared and remote medical expertise by ICT (Information Communication Technology), no matter where the patient or relevant information is located". The authors regard telemedicine as a part of health telematics-their definition coming from the European project AIM (Advanced Informatics in Medicine programme, 1993). This demonstrates a technology driven definition of the term telemedicine and so the focus of the book itself is similarly technological in nature.

A major issue in ICT systems, and especially for ICT in health and medicine, is supporting communication between various partners. Delivering integrated healthcare is an area where enhanced, efficient and cheap communication between professionals, and between professionals and patients, plays a most important role. However, in medicine, the ease of use of telecommunication systems, of information sources, of feedback and integration of services is after many years of work still not at a sufficient level. Thus, books in this area are needed.

The four running titles of the sections of the book are:

1) Background; two short introductory chapters are presented to put telemedicine in perspective. Definitions are mentioned although no sharp definition is given in this section. Some technological basics about bits, bites and transmission speeds are discussed as well as some examples of (almost historical) transmission technologies.

2) Telemedicine applications; three chapters then discuss applied approaches including pre-recorded, real-time transmission, and tele-education. Simple presentations are given of the state of art of a few years ago. More recent examples-such as the American Health Hero project and some EU projects-are more complex and challenging examples.

3) How to be successful at telemedicine; this section is divided into four chapters. It is about criteria for the development of new telemedicine systems, with one chapter by Paul Taylor handling the evaluation of telemedicine systems. The chapters provide a good insight into the criteria required for setting specifications of a new telemedicine system.

4) Other aspects of telemedicine; the later chapters tackle the issues of law, ethics, information and the future.

The target readership is mentioned in the foreword as the 'tele-clinician'. This is surely the interested reader of seven years ago (at the time of the $1^{\text {st }}$ edition) since, in my opinion, the time has come that the clinician should not worry anymore about the technology (at least not at a level of bits/second and storage capacity!). Nowadays, in most cases, the technology is secure internet-based software and hardware with a critical criteria being how fast and readable a picture is; whether systems create an immediate feedback or response from and to people at the distant place; how easy it is to store and retrieve information; and what type of functions (speaking in terms of healthcare) can be achieved. It is the task of the engineer to create technical systems which provide the functionality as required by healthcare.

What this book misses is a proper attention to ergonomics. It is mentioned in a few places but not substantiated and discussed in sufficient detail. The relation of ergonomics and safety is delicate: (human) errors can be caused by wrong design of the interfaces. And we do have at least three interfaces, such as:

(a) the patient $\Leftrightarrow$ system interface with sensors, controls and displays with feedback;

(b) the clinician/care staff $\Leftrightarrow$ system interface with options for easy viewing, easy analysis (calculations, judging etc.) and easy creation of feedback messages to the patient and filing options; and

(c) the technical supporting personnel $\Leftrightarrow$ system interface for maintenance and repair as well as for updating software.

Furthermore, in the area of access to information the book seems to completely have forgotten to mention the possibilities of new technologies that communicate with the patients. When we speak about integrated care, telematic applications with patients are increasingly important, especially where the value of an active 
role of the patient in the system is shown to be important.

Furthermore, as mentioned in the chapter of "The Future Being Expected", the role of sensors as the input channel to the systems is described as challenging, but not much is discussed about the process in the book. In the case of integrated medicine and care we must be aware that several medical and care fields come together and can work together in an efficient way when there is a common framework for communication: standards can be developed for quality, presentation formats, protocols for analysis and for proposing interventions at all levels (medical, care, operational etc.).

What this book makes clear is that telemedicine must be evaluated objectively across a number of issues: user (and patient) satisfaction, medical outcome and financial/economic results. A vast amount of literature on evaluating telecare is listed and reviewed in Chapter 8 by Paul Taylor. As said previously, evaluation must be the basis for developing new and better criteria for telemedicine and the chapter is a valuable contribution. The chapter shows that much research has to be done to find a good methodology (fast results, cost effective and complete). Present methodologies are time consuming and not keeping up with the pace of developments in this area. Attention is also given in the methodological discussion that side effects can happen. And (positive) side effects could be even more interesting for the future of telemedicine.

Telemedicine will cause major opportunities for integrated care through improved communication between professionals. However, it is not yet clear what the real effects are in both quantitative and qualitative terms. Qualitatively, we might expect changes in the roles, responsibilities and behaviour between patient and clinician-an aspect that does not gain much attention in the book. In conclusion, I would say this book is too much focussed on technologies and the experiences of the past (pre-1999) and the update to the second edition is disappointing. In particular, the book falls short in discussing the importance of telemedicine for the integration of medical and care disciplines. That said, the chapter on evaluating the effectiveness of the telemedicine systems is valuable.

Mathijs Soede

Assistive Technology Programme, iRv Institute for Rehabilitation Research, Hoensbroek

The Netherlands 